\title{
Exhibition of a Lens
}

This content has been downloaded from IOPscience. Please scroll down to see the full text. 1903 Proc. Phys. Soc. London 18423

(http://iopscience.iop.org/1478-7814/18/1/339)

View the table of contents for this issue, or go to the journal homepage for more

Download details:

IP Address: 129.93.16.3

This content was downloaded on 01/10/2015 at 22:48

Please note that terms and conditions apply. 


\section{Eixhibition of a Lens. \\ $B y$ Mr. T. H. BlaresLex *.}

The lens exhibited has the property that the two conjugate foci always move with the same relative rate along the axis. The size of the object always bears to the size of the image the same ratie, so that using the same object the image is always of the same size. The instrument is of one piece of glass, and constitutes a telescope whose magnifying power is the ratio which the object bears to the image in size, linear. The relation of the rate of motion of the object to that of the image is the square of the magnifying power. The following method of construction attains these ends. Let $\mathrm{D}$ be the distance between the centres from which the faces are struck, and let $l$ be the length of the instrument between the end faces. Then $l=\mu \mathrm{D}$ where $\mu$ is the index. The quantity $l-D$ is thus fixed. Divide it into any two parts (algebraically) $r_{1}$ and $-r_{2}$, so that $r_{1}-r_{2}=l-\mathcal{D}$, and employ these two values $r_{1}$ and $r_{2}$ as the radii of the end faces. The ratio $r_{1} / r_{2}$ will be the magnifying power $m$, which must be interpreted thus :-If the curvatures are towards the same direction $r_{1}$ and $r_{2}$ have the same sign and $m$ is positive. Double convexity therefore implies an inversion of the image, or a negative magnifying power. If $x$ be the distance of the object from the first surface encountered by the light, and $y$ be the distance of the image from the second surface, both measured positively in the direction opposite to that of lightpropagation, then

from which

$$
y m^{2}-m \mathrm{D}=x \text { wher } m=\frac{r_{1}}{r_{2}},
$$

$$
\frac{d x}{d y}=m^{2} \text {. }
$$

The fundamental condition $l=\mu D$ is that which implies an infinite focal length for a lens. This condition rules in a telescope in which the principal foci of the objective and ocular coincide. The instrument exbibited has a value +5 for the magnifying power, and was convexo-concave accordingly. One view to take of the instrument is to imagine the space between an objective and ocular properly situated, filled up with glass.

* Read March 27, 1903. 